\title{
STABILITY IN FUNCTIONAL DIFFERENCE EQUATIONS USING FIXED POINT THEORY
}

\author{
YousseF N. RAFFOUL
}

\begin{abstract}
We consider a functional difference equation and use fixed point theory to analyze the stability of its zero solution. In particular, our study focuses on the nonlinear delay functional difference equation

$$
x(t+1)=a(t) g(x(t-r)) .
$$
\end{abstract}

\section{Introduction}

When dealing with nonlinear functional differential or difference equations, it is popular to use the concept of Lyapunov functionals to qualitatively analyze their behavior. However, the use of Lyapunov functionals require ingenuity in the construction of such a function and moreover, the end results heavily depend on the constructed Lyapunov functional. For the purpose of illustration we consider the nonlinear delay difference equation

$$
x(t+1)=a(t) g(x(t))+b(t) h(x(t-r)),
$$

where the functions $g$ and $h$ are continuous. Define the Lyapunov functional $V$ by

$$
V(t)=|x(t)|+\sum_{s=t-r}^{t-1}|b(s+r)||h(x(s))| .
$$

We assume that there are positive constants $\gamma_{1}$ and $\gamma_{2}$ such that $|g(x)| \leq \gamma_{1}|x|$ and $|h(x)| \leq \gamma_{2}|x|$, so that

$$
\gamma_{1}|a(t)|+\gamma_{2}|b(t)|-1 \leq-\beta, \beta>0 .
$$

Then along solutions of (1.2) we have

$$
\begin{aligned}
\triangle V= & |x(t+1)|-|x(t)|+|b(t+r)||h(x(t))|-|b(t)||h(x(t-r))| \\
\leq & |a(t)||g(x(t))|+|b(t)||h(x(t-r))|-|x(t)| \\
& +|b(t+r)||h(x(t))|-|b(t)||h(x(t-r))|
\end{aligned}
$$

Received October 31, 2012; Revised September 29, 2013.

2010 Mathematics Subject Classification. 39A10, 39A11, 39A12.

Key words and phrases. completely delayed, discrete, fixed point, Lyapunov functionals, contraction mapping. 


$$
\leq\left(\gamma_{1}|a(t)|+\gamma_{2}|b(t)|-1\right)|x(t)| \leq-\beta|x(t)| .
$$

Now one might consult [8] and argue that the zero solution of (1.1) is asymptotically stable. For more on the use of Lyapunov functional we ask the reader to consult with [1], [2], [3], [6], [12], [13], [15]. For more recent results on the existence of periodic solutions in difference equations we refer the reader to [4], [7], [5], [9], and [14]. We remark that it is difficult if not possible to construct a suitable Lyapunov functional for (1.2) to study it stability. That is due to the absence of the term $a(t)$ as in (1.1).

In this paper we limit our study to highly nonlinear delay difference equation, typified by

$$
x(t+1)=a(t) g(x(t-r)),
$$

where $a(t): \mathbb{Z}^{+} \rightarrow \mathbb{R}$ and $r$ is a positive integer. More conditions on $g$ are forthcoming. In the paper of Raffoul [10], the author considered the linear difference equation

$$
\triangle x(t)=-a(t) x(t-r)
$$

and used fixed point theory and obtained asymptotic and periodicity results using fixed point theory. It is worth mentioning here that our equation (1.2) has fundamental difference from the above mentioned equation due to the nonlinearity that the function $g$ presents. Moreover, when inverting (1.2) in order to construct a mapping that is suitable for fixed point theory one will have to introduce a linear term which results in the addition term of $x-g(x)$. Also, the results of this paper offer the use of nonconventional metric in order to avoid that the contraction constant not to depend on the Lipschitz constant $K$ that $g$ will be required to satisfy. Recently, thee author used Lyapunov functional and obtained conditions that guaranteed the exponential asymptotic stability of the zero solution of (1.3). Also in the paper, conditions were given for the instability of the zero solution of (1.3).

\section{Stability}

First we rewrite (1.2) and have it ready to for inversion so that fixed point theory can be used. Rewrite (1.2) as

$$
x(t+1)=a(t+r) g(x(t))-\triangle_{t} \sum_{s=t-r}^{t-1} a(s+r) g(x(s)),
$$

where $\triangle_{t}$ represents that the difference is with respect to $t$. We must create a linear tern in $x$ in order to be able to invert. Thus, we add and subtract $a(t+r) x(t)$ and get,

$(2.1) x(t+1)=a(t+r) x(t)-a(t+r)[x(t)-g(x(t))]-\triangle_{t} \sum_{s=t-r}^{t-1} a(s+r) g(x(s))$. 
For each $t_{0} \geq 0$, the equation (2.1) requires initial function $\psi:\left[t_{0}-r, t_{0}\right] \rightarrow \mathbb{R}$ in order to specify a solution $x\left(t, t_{0}, \psi\right)$. The computation are the same for any $t_{0} \geq 0$ and so we take $t_{0}=0$. Thus, we say $x(t):=x(t, 0, \psi)$ is a solution of (2.1) if $x(t)=\psi(t)$ on $[-r, 0]$ and $x(t)$ satisfies (2.1) for $t \geq 0$. We begin with the following lemma which we omit its proof.

Lemma 2.1. Suppose that $a(t+r) \neq 0$ for all $t \in \mathbb{Z}^{+}$. Then $x(t)$ is a solution of the equation (2.1) if and only if

$$
\begin{aligned}
x(t)= & \psi(0) \prod_{s=0}^{t-1} a(s+r)-\sum_{s=t-r}^{t-1} a(s+r) g(x(s)) \\
& +\prod_{u=0}^{t-1} a(u+r) \sum_{s=-r}^{-1} a(s+r) g(\psi(s)) \\
& +\sum_{s=0}^{t-1}\left(a(s+r) \prod_{k=s+1}^{t-1} a(k+r) \sum_{u=s-r}^{s-1} a(u+r) g(x(u))\right) \\
& -\sum_{s=0}^{t-1}\left(\prod_{u=s+1}^{t-1} a(u+r)\right) a(s+r)[x(s)-g(x(s))], t \geq 0 .
\end{aligned}
$$

Throughout this paper it is assumed that the function $g$ is continuous, locally Lipschitz with Lipschitz constant $K$ and odd. On the other hand, we assume that $x-g(x)$ is nondecreasing and $g(x)$ is increasing on an interval $[0, L]$ for some $L>0$. Due to these assumptions, it is obvious that the functions $g(x)$ and $x-g(x)$ are locally Lipschitz with the same Lipschitz constant $K>0$.

Note that if $0<L_{1}<L$, then the conditions on $g$ hold on $\left[-L_{1}, L_{1}\right]$. Also note that if $\phi:[-r, \infty) \rightarrow \mathbb{R}$ with $\phi_{0}=\psi$, and if $|\phi(t)| \leq L$, then for $t \geq 0$ we have

$$
|\phi(t)-g(\phi(t))| \leq L-g(L),
$$

since $x-g(x)$ is odd and nondecreasing on $[0, L]$. Here $\phi_{0}=\psi(s)$ for $-r \leq s \leq 0$. The proof of Lemma 2.1 follows easily from the variation of parameters formula followed by summation by parts.

Next we define what it means for a sequence $x$ to be a solution of (1.1).

Let $\psi(t)$ be a given bounded initial function such that $\psi:\left[-r_{0}, t_{0}\right] \rightarrow \mathbb{R}$. It presents no difficulties to start solutions at any initial time $t_{0}$. However, we chose to start solutions at $t_{0}=0$. With this in mind, we say $x(t):=x(t, 0, \psi)$ is a solution of (1.1) if $x(t)=\psi(t)$ on $[-\tau, 0]$ and $x(t)$ satisfies (1.1) for $t \geq 0$. For any bounded initial sequence $\psi$ on $[-r, 0]$ with $|\psi(t)| \leq L$

$$
S=\left\{\phi:[-r, \infty) \rightarrow \mathbb{R}: \phi_{0}=\psi,|\phi(t)| \leq L\right\} .
$$

For $\phi \in S$, we define $P: S \rightarrow S$ by

$$
(P \phi)(t)=\psi(t) \text { if }-r \leq t \leq 0
$$


and

$$
\begin{aligned}
(P \phi)(t)= & \psi(0) \prod_{s=0}^{t-1} a(s+r)-\sum_{s=t-r}^{t-1} a(s+r) g(\phi(s)) \\
& +\prod_{u=0}^{t-1} a(u+r) \sum_{s=-r}^{-1} a(s+r) g(\psi(s)) \\
& +\sum_{s=0}^{t-1}\left(a(s+r) \prod_{k=s+1}^{t-1} a(k+r) \sum_{u=s-r}^{s-1} a(u+r) g(\phi(u))\right) \\
& -\sum_{s=0}^{t-1}\left(\prod_{u=s+1}^{t-1} a(u+r)\right) a(s+r)[\phi(s)-g(\phi(s))], t \geq 0 .
\end{aligned}
$$

Let $g$ be odd, increasing on $[0, L]$, satisfy a Lipschitz condition, and let $x-g(x)$ be nondecreasing on $[0, L]$. Suppose that if $L_{1} \in(0, L]$, then

$$
\begin{aligned}
& \left|L_{1}-g\left(L_{1}\right)\right| \max _{t \geq 0} \sum_{s=0}^{t-1}\left|\left(\prod_{u=s+1}^{t-1} a(u+r)\right) a(s+r)\right|+g\left(L_{1}\right) \sum_{s=t-r}^{t-1}|a(s+r)| \\
& +g\left(L_{1}\right) \max _{t \geq 0} \sum_{s=0}^{t-1} \mid\left(a(s+r) \prod_{k=s+1}^{t-1} a(k+r)\left|\sum_{u=s-r}^{s-1}\right| a(u+r) \mid<L_{1} .\right.
\end{aligned}
$$

For the purpose of the next theorem, we note that since $g(x)$ is Lipschitz with Lipschitz constant $K$ and $g(0)=0$, then $|g(x)| \leq K|x|$.

Theorem 2.2. Let $g$ be odd, increasing on $[0, L]$, satisfy a Lipschitz condition, and let $x-g(x)$ be nondecreasing on $[0, L]$. Suppose that $a(t+r) \neq 0$ for all $t \in \mathbb{Z}^{+}$. If (2.4) hold, then every solution $x(t, 0, \psi)$ of (2.1) with small initial function $\psi(t)$, is bounded provided $P$ is a contraction.

Proof. Let $\phi \in S$. Then, by (2.4), there exists an $\alpha \in(0,1)$ such that for $t \geq 0$ then

$$
\begin{aligned}
& |(P \phi)(t)| \\
\leq & || \psi \||| \prod_{s=0}^{t-1} a(s+r)|+| \prod_{u=0}^{t-1} a(u+r)|| \mid g\left(\psi(s)|| \sum_{s=-r}^{-1}|a(s+r)|\right. \\
& +|L-g(L)| \max _{t \geq 0} \sum_{s=0}^{t-1}\left|\left(\prod_{u=s+1}^{t-1} a(u+r)\right) a(s+r)\right|+g(L) \sum_{s=t-r}^{t-1}|a(s+r)| \\
& +g(L) \max _{t \geq 0}^{t-1} \sum_{s=0}^{t-1} \mid\left(a(s+r) \prod_{k=s+1}^{t-1} a(k+r)\left|\sum_{u=s-r}^{s-1}\right| a(u+r) \mid\right. \\
\leq & || \psi|| \prod_{s=0}^{t-1}|a(s+r)|+\left|\prod_{u=0}^{t-1} a(u+r)\right||| g\left(\psi(s)|| \sum_{s=-r}^{-1}|a(s+r)|+\alpha L\right.
\end{aligned}
$$




$$
\leq \prod_{s=0}^{t-1}|a(s+r)|[|| \psi\|+K\| \psi \|] \sum_{s=-r}^{-1}|a(s+r)|+\alpha L .
$$

If we choose the initial function $\psi$ small enough so that we have

$$
\prod_{s=0}^{t-1}|a(s+r)|[\|\psi\|+K\|\psi\|] \sum_{s=-r}^{-1}|a(s+r)|<(1-\alpha) L,
$$

then we will have

$$
|(P \phi)(t)| \leq(1-\alpha) L+\alpha L=L .
$$

Thus, $P: S \rightarrow S$. This shows that any solution $x(t, 0, \psi)$ of $(2.1)$ that is in $S$ is bounded. Next we show that $P$ defines a contraction map. Using the regular maximum norm, will require that the contraction constant to depend on the Lipschitz constant $K$. Instead we use the weighted norm $|\cdot|_{K}$ where for $\phi \in S$, we have

$$
|\phi|_{K}=\max _{t \geq 0}\left|\frac{1}{d K} \prod_{s=0}^{t-1}\right| a(s+r) \phi \mid \text { for } d>0 .
$$

Proposition 2.3. Let $g$ be odd, increasing on $[0, L]$, satisfy a Lipschitz condition, and let $x-g(x)$ be nondecreasing on $[0, L]$. Suppose that $a(t+r) \neq 0$ for all $t \in \mathbb{Z}^{+}$with $|a(t+r)| \leq \frac{1}{2}$. Then $P$ is a contraction with contraction constant $d>3$.

Proof. Let $\phi, \varphi \in S$. Then for $t \geq 0$, we have

$$
\begin{aligned}
& |(P \phi)-(P \varphi)|_{K} \\
\leq & \sum_{s=t-r}^{t-1}|a(s+r)||g(\phi(s))-g(\varphi(s))|\left|\frac{1}{d K} \prod_{u=0}^{t-1}\right| a(u+r) \mid \\
& +\sum_{s=0}^{t-1}\left|a(s+r) \prod_{k=s+1}^{t-1} a(k+r)\right| \sum_{u=s-r}^{s-1}|a(u+r)||g(\phi(s))-g(\varphi(s))|\left|\frac{1}{d K} \prod_{u=0}^{t-1}\right| a(u+r) \mid \\
& +\sum_{s=0}^{t-1}\left(\prod_{u=s+1}^{t-1} \mid a(u+r)\right)|| a(s+r)|| \phi(s)-g(\phi(s))-(\varphi(s)-g(\varphi(s)))|| \frac{1}{d K} \prod_{u=0}^{t-1}|a(u+r)| .
\end{aligned}
$$

Our aim is to simplify (2.6). First we remind the reader that due to the conditions on $g(x)$ and $x-g(x)$, both functions share the same Lipschitz constant $K$. Moreover, since $|a(t+r)| \leq \frac{1}{2}$, we have $|a(t+r)| \leq 1-|a(t+r)|$ and $|a(t+r)|^{2} \leq 1-|a(t+r)|^{2}$. Next, we consider the first term of (2.6)

$$
\begin{aligned}
& \sum_{s=t-r}^{t-1}|a(s+r)| \mid g(\phi(s))-g\left(\varphi(s)|| \frac{1}{d K} \prod_{u=0}^{t-1}|a(u+r)|\right. \\
\leq & \max _{t \geq 0} \frac{K}{d K} \sum_{s=t-r}^{t-1}|a(s+r)||\phi(s)-\varphi(s)| \prod_{u=0}^{s-1}|a(u+r)| \prod_{u=s}^{t-1}|a(u+r)|
\end{aligned}
$$




$$
\begin{aligned}
& \leq \frac{1}{d}|\phi-\varphi|_{K} \max _{t \geq 0} \sum_{s=t-r}^{t-1}|a(s+r)| \prod_{u=s}^{t-1}|a(u+r)| \\
& \leq \frac{1}{d}|\phi-\varphi|_{K} \max _{t \geq 0} \sum_{s=t-r}^{t-1}|a(s+r)| \prod_{u=s+1}^{t-1}|a(u+r)| \\
& \leq \frac{1}{d}|\phi-\varphi|_{K} \max _{t \geq 0} \sum_{s=t-r}^{t-1}(1-|a(s+r)|) \prod_{u=s+1}^{t-1}|a(u+r)| \\
& =\frac{1}{d}|\phi-\varphi|_{K} \max _{t \geq 0} \sum_{s=t-r}^{t-1} \triangle_{s}\left(\prod_{u=s}^{t-1}|a(u+r)|\right) \\
& =\frac{1}{d}|\phi-\varphi|_{K} \max _{t \geq 0}\left(1-\prod_{u=t-r}^{t-1}|a(u+r)|\right) \\
& \leq \frac{1}{d}|\phi-\varphi|_{K} .
\end{aligned}
$$

Next we turn our attention to the second term of (2.6).

$$
\begin{aligned}
\text { (2.8) } & \sum_{s=0}^{t-1}\left|a(s+r) \prod_{k=s+1}^{t-1} a(k+r)\right| \\
& \cdot \sum_{u=s-r}^{s-1}|a(u+r)||g(\phi(s))-g(\varphi(s))|\left|\frac{1}{d K} \prod_{l=0}^{t-1}\right| a(l+r) \mid \\
\leq & \frac{1}{d}|\phi-\varphi|_{K} \max _{t \geq 0} \sum_{s=0}^{t-1}\left|a(s+r) \prod_{k=s+1}^{t-1} a(k+r)\right| \\
\leq & \frac{1}{d}|\phi-\varphi|_{K} \max _{t \geq 0} \sum_{s=0}^{t-1}|a(s+r)| \prod_{k=s+1}^{t-1} a(k+r) \mid \\
& \sum_{u=s-r}\left(1-|a(u+r)||g(\phi(s))-g(\varphi(s))|\left|\frac{1}{d K} \prod_{l=u+1}^{t-1}\right| a(l+r) \mid\right. \\
= & \frac{1}{d}|\phi-\varphi|_{K} \max _{t \geq 0} \sum_{s=0}^{t-1}|a(s+r)| \prod_{k=s+1}^{t-1} a(k+r) \mid \sum_{u=s-r}^{t-1} \prod_{s}\left(\prod_{l=u}^{t-1}|a(l+r)|\right) \\
\leq & \frac{1}{d}|\phi-\varphi|_{K} \max _{t \geq 0} \sum_{s=0}^{t-1}|a(s+r)| \prod_{k=s+1}^{t-1} a(k+r)\left|\prod_{l=s}^{t-1}\right| a(l+r) \mid
\end{aligned}
$$




$$
\begin{aligned}
& =\frac{1}{d}|\phi-\varphi|_{K} \max _{t \geq 0} \sum_{s=0}^{t-1}|a(s+r)|^{2}\left(\prod_{k=s+1}^{t-1}|a(k+r)|\right)^{2} \\
& \leq \frac{1}{d}|\phi-\varphi|_{K} \max _{t \geq 0} \sum_{s=0}^{t-1} \mid\left(1-|a(s+r)|^{2}\right)\left(\prod_{k=s+1}^{t-1}|a(k+r)|\right)^{2} \\
& \leq \frac{1}{d}|\phi-\varphi|_{K} \max _{t \geq 0} \sum_{s=0}^{t-1}\left|\triangle_{s}\left(\prod_{k=s}^{t-1}|a(k+r)|\right)^{2} \leq \frac{1}{d}\right| \phi-\left.\varphi\right|_{K} .
\end{aligned}
$$

Now we deal with the last term of (2.6).

$$
\begin{aligned}
& \sum_{s=0}^{t-1}\left(\prod_{u=s+1}^{t-1} \mid a(u+r)\right)|| a(s+r)|| \phi(s)-g(\phi(s))-(\varphi(s)-g(\varphi(s)))|| \frac{1}{d K} \prod_{u=0}^{t-1}|a(u+r)| \\
\leq & \frac{1}{d}|\phi-\varphi|_{K} \max _{t \geq 0} \sum_{s=0}^{t-1} \prod_{u=s+1}^{t-1}|a(u+r)||a(s+r)| \prod_{u=s}^{t-1}|a(u+r)| \\
\leq & \frac{1}{d}|\phi-\varphi|_{K} \max _{t \geq 0} \sum_{s=0}^{t-1}|a(s+r)|^{2}\left(\prod_{u=s+1}^{t-1}|a(u+r)|\right)^{2} \\
\leq & \frac{1}{d}|\phi-\varphi|_{K} \max _{t \geq 0} \sum_{s=0}^{t-1}\left(1-|a(s+r)|^{2}\right)\left(\prod_{u=s+1}^{t-1}|a(u+r)|\right)^{2} \\
= & \frac{1}{d}|\phi-\varphi|_{K} \max _{t \geq 0} \sum_{s=0}^{t-1} \triangle_{s}\left(\prod_{u=s}^{t-1}|a(u+r)|\right)^{2} \\
= & \frac{1}{d}|\phi-\varphi|_{K}\left(1-\left(\prod_{u=0}^{t-1}|a(u+r)|\right)^{2}\right) \\
\leq & \frac{1}{d}|\phi-\varphi|_{K} .
\end{aligned}
$$

A substitution of (2.7), (2.8) and (2.9) into (2.6) yield to

$$
|(P \phi)-(P \varphi)|_{K} \leq\left(\frac{1}{d}+\frac{1}{d}+\frac{1}{d}\right)|\phi-\varphi|_{K},
$$

which makes $P$ a contraction for $d>3$. Let $(\mathcal{X},|\cdot|)$ be the Banach space of bounded sequences $\phi:[0, \infty) \rightarrow \mathbb{R}$. As $S$ is a subset of the Banach space $\mathcal{X}$ and $S$ is closed and bounded so $S$ is complete. Thus, $P: S \rightarrow S$ has a unique fixed point. This completes the proof.

We have the following corollary.

Corollary 2.4. Let $g$ be odd, increasing on $[0, L]$, satisfy a Lipschitz condition, and let $x-g(x)$ be nondecreasing on $[0, L]$. Suppose that $a(t+r) \neq 0$ for all $t \in \mathbb{Z}^{+}$. If (2.4) hold with $|a(t+r)| \leq \frac{1}{2}$, then the unique solution $x(t, 0, \psi)$ 
of (2.1) with small initial function $\psi(t)$, is is bounded and its zero solution is stable.

Proof. Let $P$ be defined by (2.3). Then by Theorem 2.2, $P$ maps $S$ into $S$. Moreover, by Proposition $2.4 P$ is a contraction on $S$ and hence the unique solution of (2.1) is bounded by Theorem 2.2. Left to show the zero solution is stable. Let $L$ be given by (2.5) and set $0<\epsilon<L$. Choose $\delta=\frac{\epsilon(1-\alpha)}{(1+K) \prod_{s=0}^{t-1}|a(s+r)| \sum_{s=-r}^{-1}|a(s+r)|}$. Then for $|\psi|<\delta$, we have by $(2.5)$ that

$$
\begin{aligned}
|(P \phi)(t)| & \leq \prod_{s=0}^{t-1}|a(s+r)|[|| \psi||+K|| \psi||] \sum_{s=-r}^{-1}|a(s+r)|+\alpha L \\
& \leq \delta(1+K) \prod_{s=0}^{t-1}|a(s+r)| \sum_{s=-r}^{-1}|a(s+r)|+\alpha L \\
& \leq \delta(1+K) \prod_{s=0}^{t-1}|a(s+r)| \sum_{s=-r}^{-1}|a(s+r)|+\alpha \epsilon \\
& \leq \epsilon(1-\alpha)+\alpha \epsilon=\epsilon .
\end{aligned}
$$

Hence the zero solution is stable. This completes the proof.

We mention here that the requirement $|a(t+s)| \leq 1 / 2$ was necessitated by the use of the norm $|\cdot|_{K}$. However, in proving that $P$ is a contraction we did not have to involve $K$ in the contraction constant.

Example. Let $a(t+r) \neq 0$ such that $|a(t+r)| \leq \frac{1}{2}$. Consider

$$
x(t+1)=-a(t) x^{3}(t-r) .
$$

In view of (2.1) we have

$$
x(t+1)=a(t+r) x(t)-a(t+r)\left[x(t)-x^{3}(t-r)\right]+\triangle_{t} \sum_{s=t-r}^{t-1} a(s+r) x^{3}(s) .
$$

Let $f(x)=x-x^{3}$. Then $f(x)$ is increasing on $\left(0, \frac{1}{\sqrt{3}}\right)$ and has a maximum of $\frac{2}{3 \sqrt{3}}$ at $x=\frac{1}{\sqrt{3}}$. For any bounded initial sequence $\psi$ on $[-r, 0]$ with $|\psi(t)| \leq \frac{1}{\sqrt{3}}$ we set

$$
S=\left\{\phi:[-r, \infty) \rightarrow \mathbb{R}: \phi_{0}=\psi,|\phi(t)| \leq \frac{1}{\sqrt{3}}\right\} .
$$

For $\phi \in S$, we define $P: S \rightarrow S$ by

$$
(P \phi)(t)=\psi(t) \text { if }-r \leq t \leq 0
$$

and

$$
(P \phi)(t)=\psi(0) \prod_{s=0}^{t-1} a(s+r)+\sum_{s=t-r}^{t-1} a(s+r) \phi^{3}(s)
$$




$$
\begin{aligned}
& -\prod_{u=0}^{t-1} a(u+r) \sum_{s=-r}^{-1} a(s+r) \psi^{3}(s) \\
& -\sum_{s=0}^{t-1}\left(a(s+r) \prod_{k=s+1}^{t-1} a(k+r) \sum_{u=s-r}^{s-1} a(u+r) \phi^{3}(u)\right) \\
& -\sum_{s=0}^{t-1}\left(\prod_{u=s+1}^{t-1} a(u+r)\right) a(s+r)\left[\phi(s)-\phi^{3}(s)\right], t \geq 0
\end{aligned}
$$

Let $\psi$ be small enough so that

$$
\begin{aligned}
& \|\psi\| \prod_{s=0}^{t-1}|a(s+r)|+\frac{\sqrt{3}}{9} \sum_{s=t-r}^{t-1}|a(s+r)|+|| \psi \|\left|\prod_{u=0}^{t-1}\right| a(u+r)\left|\sum_{s=-r}^{-1}\right| a(s+r) \mid \\
& +\frac{\sqrt{3}}{9} \sum_{s=0}^{t-1}\left(|a(s+r)| \prod_{k=s+1}^{t-1}|a(k+r)| \sum_{u=s-r}^{s-1}|a(u+r)|\right) \\
& +\frac{2}{3 \sqrt{3}} \sum_{s=0}^{t-1}\left(\prod_{u=s+1}^{t-1} \mid a(u+r)\right)|| a(s+r) \mid \leq \frac{1}{\sqrt{3}}
\end{aligned}
$$

Then

$$
|(P \phi)(t)| \leq \frac{1}{\sqrt{3}} .
$$

Moreover, it is obvious that the Lipscihtz constant $k=1$. Let $d$ be a positive constant such that $d>3$. Then if we take

$$
|\phi|_{1}=\max _{t \geq 0}\left|\frac{1}{d} \prod_{s=0}^{t-1}\right| a(s+r) \phi \mid,
$$

we have $P$ is contraction on $S$ and hence all solutions of (2.10) are bounded and its zero solution is stable.

\section{References}

[1] J. Cermák, Difference equations in the qualitative theory of delay differential equations, Proceedings of the Sixth International Conference on Difference Equations, 391-398, CRC, Boca Raton, FL, 2004.

[2] S. Elaydi, An Introduction to Difference Equations, Springer, New York, 1999.

[3] W. Kelley and A. Peterson, Difference Equations, Academic Press, 2001.

[4] W.-T. Li and H.-F. Huo, Positive periodic solutions of delay difference equations and applications in population dynamics, J. Comput. Appl. Math. 176 (2005), no. 2, 357369.

[5] M. Maroun and Y. N. Raffoul, Periodic solutions in nonlinear neutral difference equations with functional delay, J. Korean Math. Soc. 42 (2005), no. 2, 255-268.

[6] J. Migda, Asymptotic behavior of solutions of nonlinear difference equations, Math. Bohem. 129 (2004), no. 4, 349-359.

[7] M. Islam and E. Yankson, Boundedness and Stability For Nonlinear Delay Difference Equations Employing Fixed Point Theory, in review. 
[8] Y. Raffoul, General theorems for stability and boundedness for nonlinear functional discrete systems, J. Math. Anal. Appl. 279 (2003), no. 2, 639-650.

[9] _ Periodicity in general delay non-linear difference equations using fixed point theory, J. Difference Equ. Appl. 10 (2004), no. 13-15, 1229-1242.

[10] $ـ$ Stability and periodicity in discrete delayed equations, J. Math. Anal. Appl. 324 (2006), no. 2, 1356-1362

[11] - Inequalities That Lead To Exponential Stability And Instability In Delay Difference Equations, J. Inequal. Pure Appl. Math. 10 (2009), no. 3, Article 70, 9pp.

[12] C. Qian and Y. Sun, On global attractivity of nonlinear delay difference equations with a forcing term, J. Difference Equ. Appl. 11 (2005), no. 3, 227-243.

[13] D. C. Zhang and B. Shi, Global behavior of solutions of a nonlinear difference equation, Appl. Math. Comput. 159 (2004), no. 1, 29-35.

[14] G. Zhang and L. Zhang, Periodicity and attractivity of a nonlinear higher order difference equation, Appl. Math. Comput. 161 (2005), no. 2, 395-401.

[15] H. Zhu and L. Huang, Asymptotic behavior of solutions for a class of delay difference equation, Ann. Differential Equations 21 (2005), no. 1, 99-105.

Department of Mathematics

UNIVERSITY OF DAYTON

DAYTON, OH 45469-2316, USA

E-mail address: yraffoul1@udayton.edu 